\title{
Development of intelligent unlocking key management system
}

\author{
Jin Huang ${ }^{1, a}$ Ningkai Shi ${ }^{2, b}$ \\ ${ }^{1}$ NARI Technology Development Co., Ltd. Nanjing, 211106, China \\ ${ }^{2}$ NARI Technology Development Co., Ltd. Nanjing, 211106, China \\ ahuangjin210203@126.com bsnk81@qq.com
}

Keywords: anti-misoperation system of power, unlocking key management, web server

Abstract. In the electric power system, the traditional unlocking key management method has many drawbacks, such as inconvenient examination and authorization, troubled operation, inaccurate records. An intelligent unlocking key management system is presented, which is based on network centralized control. This system can record who and when use which unlocking key, designate unlocking area through network. System principle, hardware configuration, embedded software and server software are designed.

\section{Introduction}

In the field of power systems, we use computer technology to ensure the safety of personal and equipment. Related devices have been developing rapidly over the past decade. However, when the device fails, the original manual approval management model can't guarantee the specification of the implementation of unlock operations, but also increase the workload of managers. In order to eliminate the illegal work of personnel, random unlock caused by the occurrence of misuse of the accident, the urgent need to provide technically unlock the operation of effective management measures. So far, although many companies engaged in computer anti-misoperation system research, developed an unlocking key management machine to control the unlocking key access. However, with the centralized control operation mode into the field of substation management, the original unlocking key management unit of the single-station management mode, cannot be well adapted to the requirements of on-site security operations. To solve this problem, we propose a new type of intelligent unlocking key management system based on Ethernet control and management, which can define unlock range. The successful application of this system will greatly improve the operation mode of the centralized control of the transformer substation safety factor and efficiency.

The basic structure of centralized control unlocking key management system includes WEB server, switch, intelligent unlocking key management devices. Fig 1 shows network of unlocking key management system. The Web server manages all the intelligent unlocking key management devices connected to the LAN, and can view the unlocking key usage in each management device in real time and analyze and statistic the relevant data. 


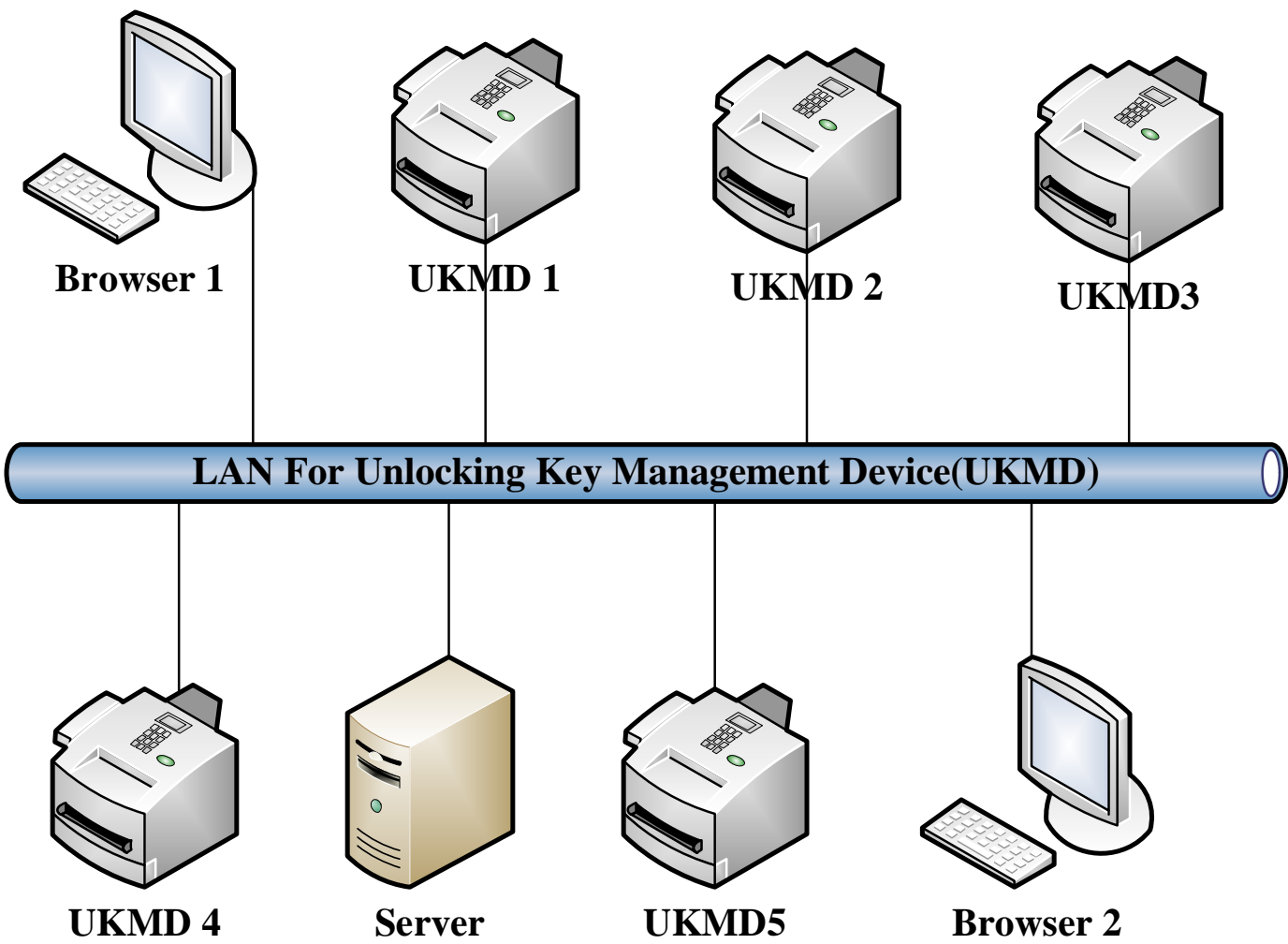

Fig. 1 network of the unlocking key management system

\section{Intelligent unlocking key management device hardware system structure}

Hardware theory. The intelligent unlocking key management device adopts the ARM9 core processor to store the intelligent unlocking key and the universal unlocking key in the two key cabin separately for management. The intelligent unlocking key storage module has an infrared data communication module for the intelligent unlocking key and the management machine communication. Universal Unlocking Keys Each slot in the key compartment also has an infrared detection circuit to check its use in real time. The device uses RFID technology to manage users with different rights. In order to achieve closed-loop control, infrared detection feedback electromagnetic lock mechanism of the door lock situation. Application of Gigabit Ethernet for data exchange so that administrators can not only easily open the release of votes will be transmitted to each management device in a timely manner to unlock operations, but also to facilitate the management of the maintenance, management, analysis and statistics of various operating information.

Circuit configuration. Intelligent unlocking key management device using ATMEL's ARM9 microprocessor AT91SAM9260 as the core, with 8MB NOR Flash, 64MB NAND Flash and 64MB SDRAM constitute the minimum target system. Fig. 2 depicts the hardware composition of the intelligent unlocking key management device. 


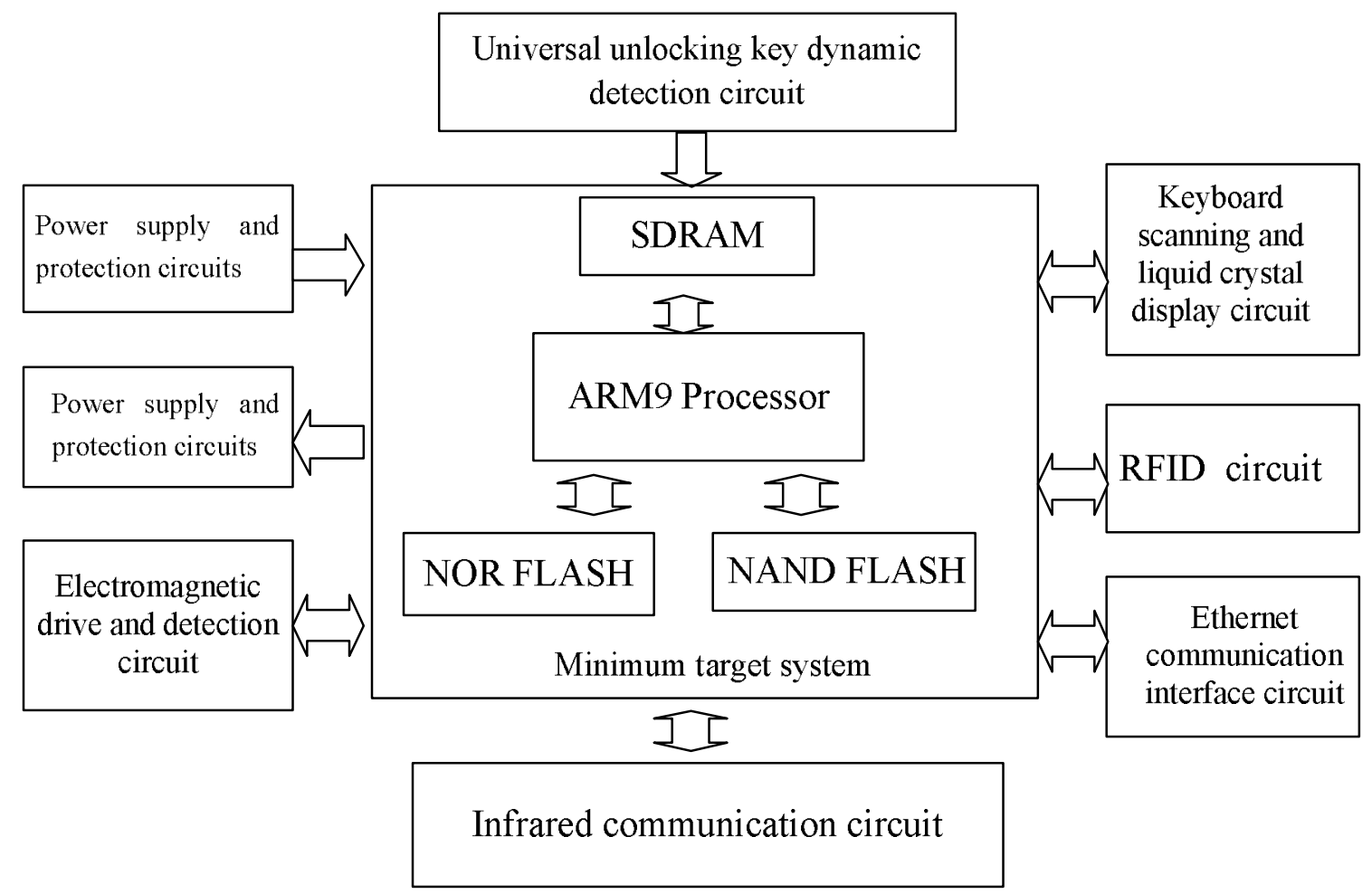

Fig. 2 hardware composition of the intelligent unlocking key management device

Infrared Detection Technology. A major highlight of the device design is the use of infrared light dynamic detection technology. Detection unit using a wavelength of 940nm infrared emission tube IRA204 and the wavelength of the most sensitive infrared receiver tube PT204 form a pair of optical detectors. When the universal unlock key inserted in the slot, IRA204 infrared light will be blocked, then PT204 cannot receive the former issued by the infrared light, and a high-level signal is outputted. At the same time, the CPU knows that the universal unlock key is in place. The advantage of using infrared light detection technology compared to the mechanical contact initiation level signal is that there is no level jitter in the course of mechanical contact, which improves the reliability of the inspection; there is no mechanical contact during wear and prolongs system life.

Mechanical implementation and testing agencies. Mechanical movement caused by infrared light changes, and then this process into a signal change, real-time transmission to the CPU, the system closed-loop control, this is another major feature of the system design. The intelligent unlocking key management device locks the door of the universal unlocking key storage compartment by an electromagnetic lock. Whether the electromagnetic lock is activated and whether the door is closed directly affects the safety using of the universal unlocking key and the accuracy of the management device to record the event. Therefore, the electromagnetic lock and door movement detection is particularly important. The management device to detect the electromagnetic lock pin action is through the reflection type infrared photoelectric detection switch GP2S04 to complete. When the pin pops up and close to the focus of GP2S04, the reflected light flux surge, infrared photoelectric switch is turned on, a low level signal is output. On the other hand, GP2S04 outputs a high level signal to the CPU. CPU to determine whether the door is closed is based on whether the lock on the door to block the infrared emission tube to receive the infrared tube. Its principle is the same as the infrared detection method of the universal unlocking key. 


\section{System software design}

The Embedded Software Design of the Intelligent Unlocking Key Management device. Intelligent unlocking key management device embedded software design is based on ARM9 as the core of the hardware platform. Embedded Linux is a device application development platform. The whole application consists of seven tasks: human-computer interaction, mechanical control and infrared detection, user rights management, event record management, record analysis and statistics, intelligent unlock operation ticket management and Ethernet communication management. Due to space reasons, this article only on the most representative of the "intelligent unlock operation ticket management tasks," a detailed analysis.

The device supports two unlock modes: 1. Make out the unlocking operation ticket, and then use the intelligent unlocking key to release the equipment; 2. In case of emergency, the universal unlocking key compartment is authorized by the advanced user to access the key stored in it. This feature is restricted to use.

Through the management of the man-machine interface to take out the unlocking operation ticket, the operator use intelligent unlocking key to unlock the authorized device. It guarantees the uniqueness of the target, such as the wrong interval will not be able to unlock. This method effectively solves the problem that the unlocking operation is uncontrolled after the operator gets the universal unlocking key. Fig. 3 shows the flow of the intelligent unlocking operation task.

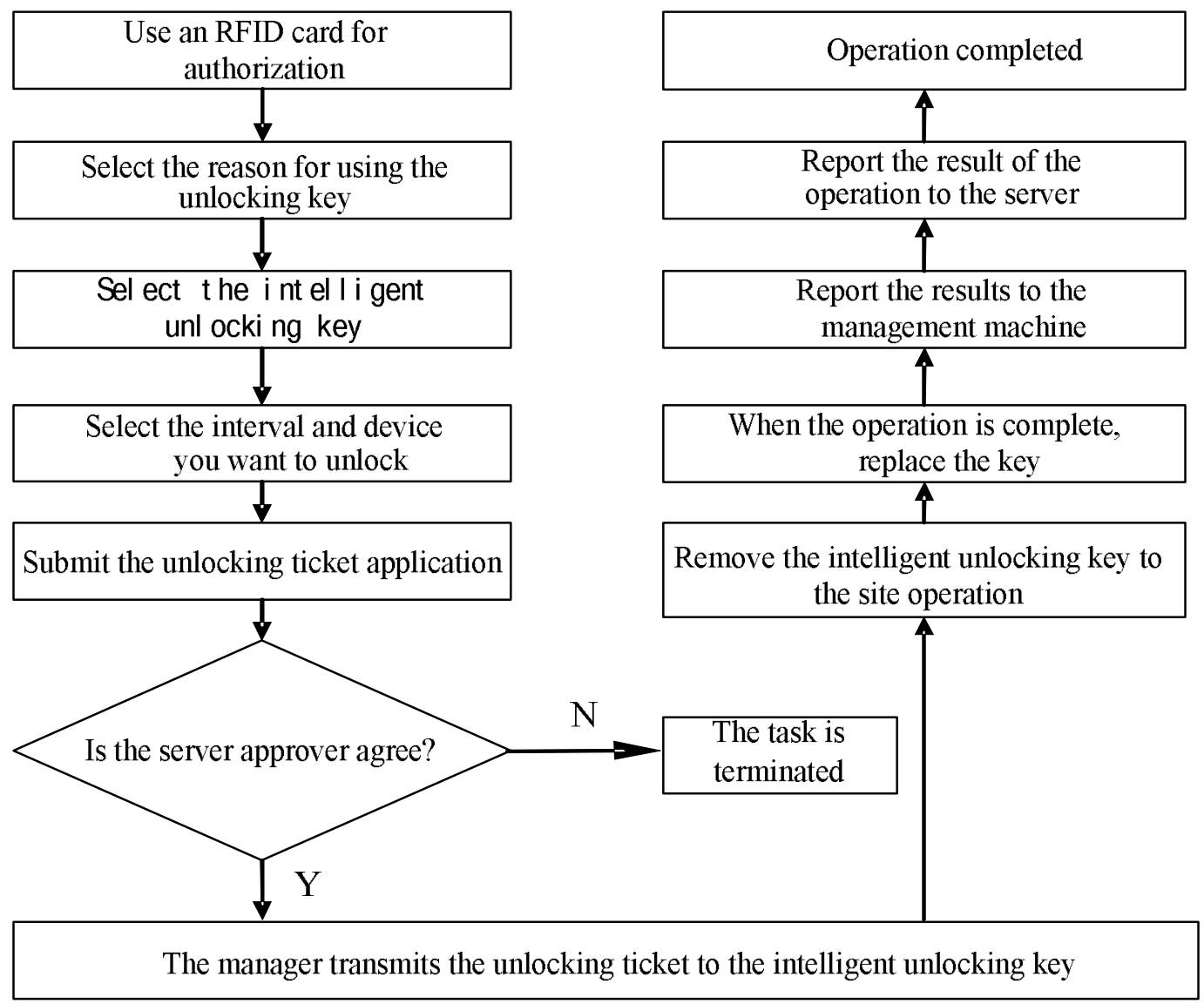

Fig. 3 The flow of the intelligent unlocking operation task

Web Server Management Software Design. Web server software is also developed on the Linux operating system. Web service dynamic browsing on Apache + Html + PHP framework program, which can dynamically modify Web Server computer system configuration. We use GCC and other development tools to develop user applications. This enables the monitoring of the usage of the 
unlocking key management device of the substation. All user interfaces are implemented through the WWW browser. Part of the transaction logic can be implemented in the unlocking management device interface, but the main transaction is implemented on the Web server. All of these form the 3-tier structure.Fig.4 shows the PHP service model structure. B/S mode is the Web Server and Browser access. In this mode, the intermediate nodes of the browser and the server do not do anything to the HTTP request and response, the application is placed in the Server. When dealing with HTTP requests, users only need to use such as IE browser on the server to browse the data, without the need to develop client programs. The system's Web Server can achieve the following functions: a. real-time view the working status of each unlocking key management device in the LAN, b. check the use of the universal unlocking key inside each management unit of the network, c. set the different levels of users, regulate the management of the unlocking key, d. Analysis and statistics the unlocking key use of each substation.

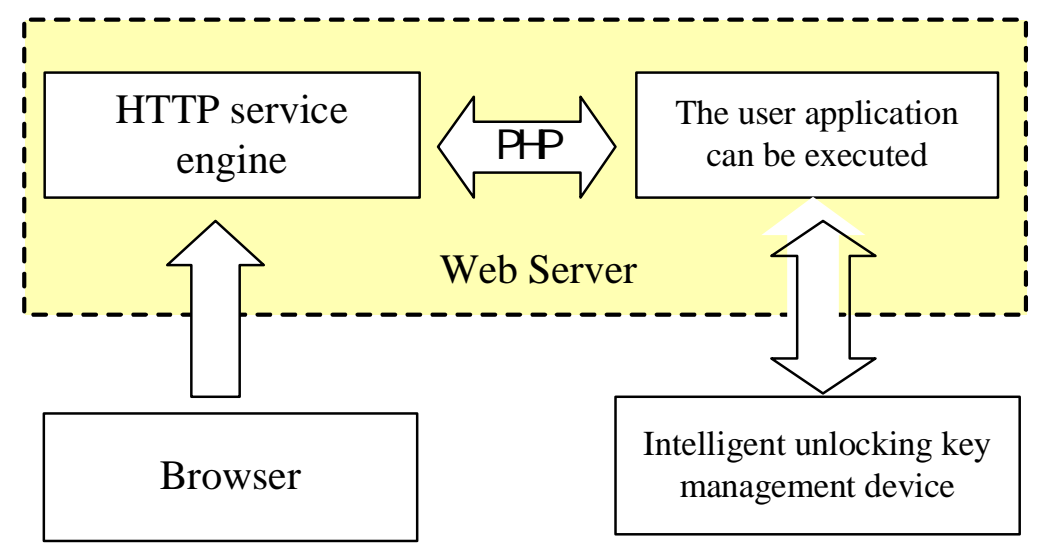

Fig. 4 PHP service model structure

\section{Conclusions}

Development of intelligent unlocking key management system adopts the embedded ARM technology and Web Server technology to realize the real-time monitoring of the unlocking key usage. The application of this system makes the management of the unlocking operation mode has undergone a new transformation. It not only ensures the safety and reliability of unlocking operation, but also reduces the workload of on-site examination and approval, and provides a new way for the whole digitization and network monitoring of power anti-misoperation.

\section{References}

[1] David Seal: ARM Architecture Reference Manual. Second Edition. Pearson Education Limited (Addsion - Wesley), 2012, p.73-76.

[2] Steve Furber: ARM System-on-Chip Architecture. Second Edition. Pearson Education Limited (Addsion - Wesley), 2014, p.68-72

[4] WEHRLE K, PAHLKE F: The Linux networking architecture [M]. America, Prentice Hall, 2010, p.92-96

[5] International Forecourt Standard Forum. Communication on specification over TCP/IP[S], version 1.00 , England, 2001.

[6] Yimin Sun, Lin Hou, Ping Jie: Implementation of mal-opration proof on platforms of relay and control units between bays. Automation of Electric Power System, 2015, 30(16), p. 81-85

[7] Renqi Liu, Xiaojun Lv, Jin Huang, Ting Su: An Integrated Temporary Grounding Line Management System. Automation of Electric Power Systems, 2010,34(22), p.109-112

[8] Information on http://www.atmel.com 